\title{
Histochemical localisation of mitochondrial enzyme activity in human optic nerve and retina
}

\author{
Richard M Andrews, Philip G Griffiths, Margaret A Johnson, Douglass M Turnbull
}

\begin{abstract}
Aims-To demonstrate the quantitative distribution of mitochondrial enzymes within the human optic nerve and retina in relation to the pathogenesis of ophthalmic disease.

Methods-Enucleations were performed at the time of multiple organ donation and the optic nerve and peripapillary retina immediately excised en bloc and frozen. Reactivities of the mitochondrial enzymes cytochrome $c$ oxidase and succinate dehydrogenase were demonstrated in serial cryostat sections using specific histochemical assays.

Results-In the optic nerve the unmyelinated prelaminar and laminar regions were rich in both cytochrome $c$ oxidase and succinate dehydrogenase. Myelination of fibres as they exited the lamina cribrosa was associated with an abrupt reduction in enzyme activity. Within the retina, high levels of enzyme activity were found localised within the retinal ganglion cells and nerve fibre layer, the outer plexiform layer, inner segments of photoreceptors, and the retinal pigment epithelium. Conclusions-Mitochondrial enzyme activity is preserved in human optic nerve and retina retrieved at the time of multiple organ donation. The distribution of enzyme activity within the eye has implications for the understanding of the pattern of ophthalmic involvement seen in mitochondrial diseases and the site of ganglion cell dysfunction in those patients with optic nerve involvement.

(Br F Ophthalmol 1999;83:231-235)
\end{abstract}

Department of Ophthalmology, University of Newcastle upon Tyne $\mathrm{R} M$ Andrews P G Griffiths

Department of Neurology, University of Newcastle upon

Tyne

M A Johnson

D M Turnbul

Correspondence to: Mr Richard Andrews, Department of Neurology,

The Medical School, Framlington Place, Newcastle upon Tyne, $\mathrm{NE} 24 \mathrm{HH}$.

Accepted for publication 1 September 1998 upon the coordinated activity of five multisubunit enzyme complexes located within the mitochondrial inner membrane. Transfer of electrons through respiratory chain complexes I-IV releases energy which is used to pump protons across the inner mitochondrial membrane at complexes I, III, and IV. This generates an electrochemical gradient across the inner membrane which is exploited by complex V (ATP synthase) to make ATP from adenosine diphosphate and inorganic phosphate. ${ }^{1}$

Mitochondrial DNA (mtDNA) encodes for some subunits of these enzyme complexes and OXPHOS defects due to mtDNA mutations are now known to cause a diverse group of human diseases. ${ }^{2}$ Although many of the under- lying molecular genetic and associated biochemical defects have now been described, the relation between these and the pattern of tissue involvement is poorly understood. Tissue specific differences are known to exist in the control of OXPHOS ${ }^{3}$ and the degree to which an individual complex can be inhibited before overall mitochondrial function is impaired varies between tissues in the rat. ${ }^{4}$ Such differences are also likely to be important in determining the pattern of organ or tissue involvement in human disease. ${ }^{4}$ Mitochondrial function in different tissues therefore needs to be investigated to unravel the relation between the primary mitochondrial defect and the clinical features. ${ }^{5}$

Characterisation of mitochondrial function is largely dependent upon specific histochemical and biochemical assays. ${ }^{67}$ In all of these, preservation of mitochondrial enzyme activity is essential. This decines rapidly post mortem limiting studies to the availability of fresh tissue samples. Skeletal muscle is the most widely studied human tissue owing to the ease with which biopsy specimens may be obtained. The eye, which is frequently involved in mitochondrial disorders, ${ }^{8}$ does not lend itself to this approach. Mitochondrial function within human ocular tissues therefore remains to be defined.

Multiple organ donation provides an opportunity for retrieving human ocular tissue immediately upon the cessation of vascular perfusion. Enucleations have been performed at the time of cross clamping of the aorta and the preservation and localisation of mitochondrial enzyme activity in fresh frozen optic nerve and retina determined by histochemical assays for cytochrome $c$ oxidase (complex IV) and succinate dehydrogenase (complex II). These enzymes are specific to mitochondria and essential for OXPHOS. While mtDNA encodes for some subunits of cytochrome $c$ oxidase, succinate dehydrogenase is composed entirely of nuclear gene products. ${ }^{2}$

\section{Materials and methods}

Approval for this study was obtained through the local research ethics committee. Five patients undergoing multiple organ retrieval were selected on the basis of having no personal or family history of ophthalmic disease and no documented hypoxic or hypotensive episode before donation. In three cases the cause of death was primary intracranial haemorrhage due to the rupture of saccular aneurysms and in two the consequence of head trauma arising from road traffic accidents. No globe used in this study showed any evidence 


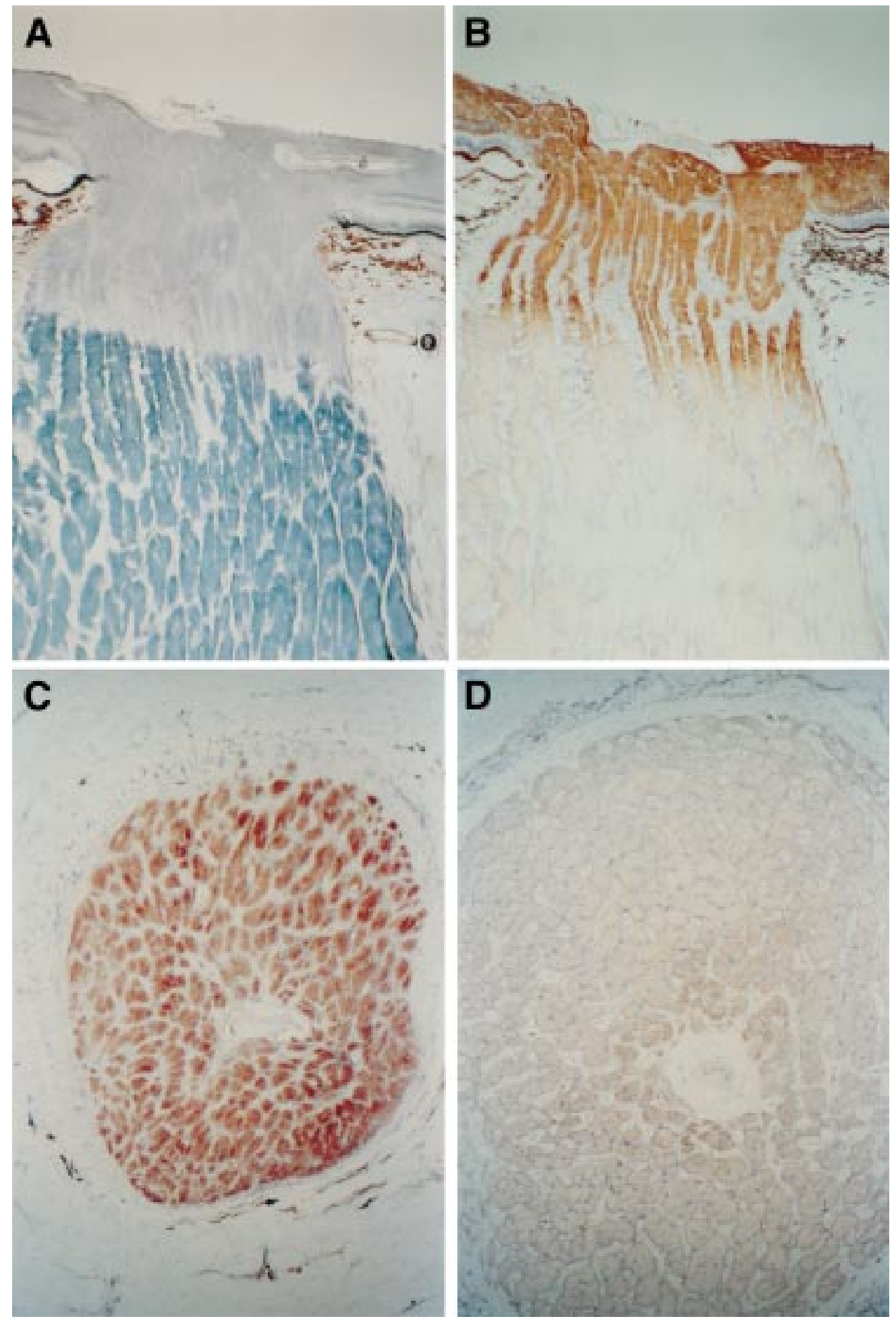

Figure 1 Photomicrographs of longitudinal sections through the optic nerve $(A)$ stained with Sudan black to demonstate myelin and $(B)$ reacted to demonstrate $C O X$ which is confined to the unmyelinated portion of retinal ganglion cell axons. Transverse sections of optic nerve $(C)$ in immediate prelaminar zone showing high COX activity and $(D)$ in the retrolaminar zone approximately $1.5 \mathrm{~mm}$ from section $(C)$ showing low COX activity.

of pathological damage on either macroscopic or subsequent microscopic examination.

Both eyes of each patient were enucleated coincident with cross clamping of the aorta and the globes placed on wet ice. Corneoscleral discs were immediately cut free and stored in culture medium (Optisol-GS, Chiron Intraoptics, Irvine, CA, USA) for transport to one of the national eye banks. The optic nerves, together with a $2-3 \mathrm{~mm}$ cuff of peripapillary tissue (sclera/choroid/retinal pigment epithelium/retina) and the overlying cortical vitreous, were then excised en bloc and frozen in isopentane cooled to $-150^{\circ} \mathrm{C}$ using liquid nitrogen. Tissue blocks were stored at $-80^{\circ} \mathrm{C}$ before sectioning.

For longitudinal sectioning, the optic nerves were supported on a $2-3 \mathrm{~mm}$ layer of gel $(10 \%$ 
gelatin $/ 30 \%$ OCT, Miles Diagnostics, Elkhart, IN, USA) to enable sectioning throughout the whole of the optic nerve/peripapillary tissue. One optic nerve of each patient was oriented transversely between two gel layers for cross sectional studies. Sections $10 \mu \mathrm{m}$ thick were cut at $-20^{\circ} \mathrm{C}$ using a Reichert $2800 \mathrm{~N}$ Frigocut cryostat microtome and mounted on chrome gelatin coated slides. Series of frozen sections, taken at multiple levels across each specimen, were stained using haematoxylin and eosin, reacted to demonstrate cytochrome $c$ oxidase $(\mathrm{COX})$ and succinate dehydrogenase $(\mathrm{SDH})$ activity, ${ }^{6}$ or stained with Sudan black in propylene glycol to demonstrate myelin. The incubation medium for COX activity contained $4 \mathrm{mM} \mathrm{3,3'-diaminobenzidine} \mathrm{hydro-}$ chloride and $100 \mu \mathrm{M}$ cytochrome $c$ in $0.1 \mathrm{M}$ phosphate, $\mathrm{pH} 7.0$, with $5 \mathrm{IU} / \mathrm{ml}$ catalase to inhibit peroxidatic activity (Sigma, Poole, Dorset). Incubation for SDH was in $130 \mathrm{mM}$ sodium succinate, $1.5 \mathrm{mM}$ nitro blue tetrazolium, $0.2 \mathrm{mM}$ phenazine methosulphate, and $1.0 \mathrm{mM}$ sodium azide in $0.1 \mathrm{M}$ phosphate, $\mathrm{pH}$ 7.0 (Sigma, Poole, Dorset). Control reactions for $\mathrm{COX}$ and $\mathrm{SDH}$ consisted of incubation without substrate and in the presence of inhibitors. ${ }^{6}$ The COX reaction, in the presence of $1 \mathrm{mM}$ cyanide or $2.5 \mathrm{mM}$ azide was virtually negative; the SDH reaction, in the absence of exogenous succinate and in the presence of the competitive inhibitor malonate (5 $\mathrm{mM}$ ), showed about $80 \%$ inhibition. Immunocytochemical (ICC) labelling of COX in optic nerve and retina was used to confirm the localisation of the enzyme protein seen in the histochemical reaction. Frozen sections $6 \mu \mathrm{m}$ thick were mounted on chrome gelatin coated slides, air dried for 2 hours, and fixed in $4 \%$
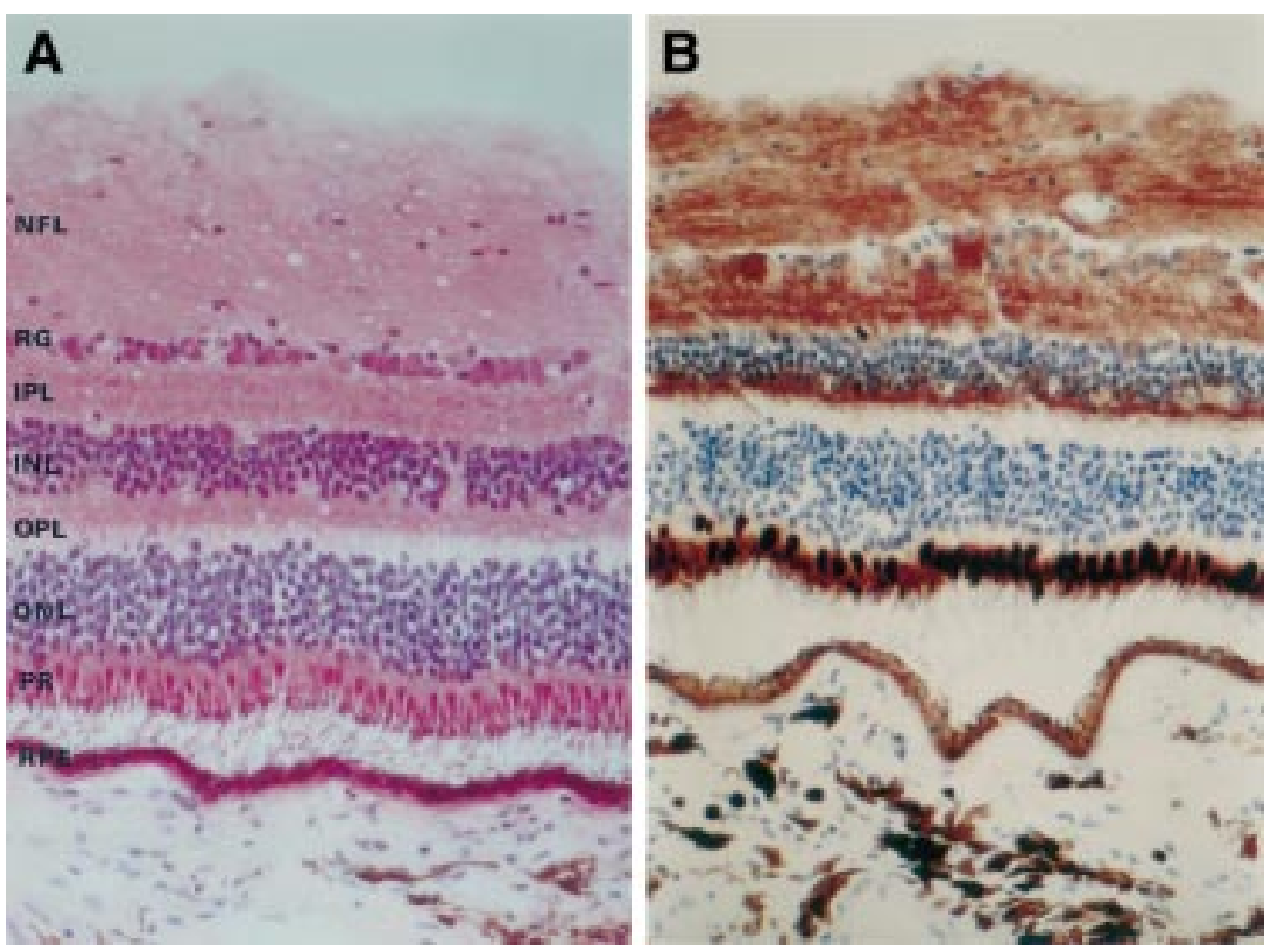

Figure 2 Photomicrographs of longitudinal sections of peripapillary retina $(A)$ stained using haematoxylin and eosin, and (B) showing the distribution and intensity of COX activity in the different retinal layers. See text for abbreviations. paraformaldehyde in $0.1 \mathrm{M}$ phosphate $\mathrm{pH} 7.4$ for 1 hour at room temperature. Sections were then permeabilised by transferring through a graded ethanol series to $100 \%$ ethanol for 15 minutes before rehydration and immunolabelling. ${ }^{9} \quad$ Monospecific antisera to $\mathrm{COX}$ subunits (rabbit polyclonals) were optimally diluted in $0.0125 \mathrm{M}$ phosphate buffered saline (PBS). Visualisation of bound antibody was by the peroxidase antiperoxidase (PAP) technique $^{10}$ using swine anti-rabbit IgG (Dako, Z196) and rabbit PAP (Dako, Z113) each diluted 1:100 in PBS.

\section{Results}

OPTIC NERVE

Preservation of anatomical integrity was shown Sudan black staining confirmed that myelination was confined to the retrolaminar portion of the optic nerve. COX activity was very low in the myelinated region but was high in the unmyelinated laminar and prelaminar portions (Fig 1). SDH showed essentially the same pattern of distribution and intensity as COX activity. This pattern was also clearly demonstrated in transverse sections (Fig 1) and consequently does not appear to be artefactual owing to a failure of penetration of the assay media through the myelin sheath. Thus, there was a clear reciprocal relation between myelination and oxidative enzyme activity in the optic nerve.

RETINA

Preservation of retinal integrity was more difficult to achieve using frozen sections as compared with conventional paraffin embedded material. However, the major histological in haematoxylin and eosin stained sections. 
layers could be clearly identified and correlated with observed variations in oxidative enzyme activity. The retinal pigment epithelium (RPE) showed both COX and SDH activity but the respective histochemical reaction products were masked to some extent by the presence of melanin granules (also present in unreacted sections) and estimation of reaction intensity was difficult. Very high COX and SDH activity was seen within the inner segments of photoreceptors (PR), particularly the cones. This contrasted with the adjacent outer nuclear layer (ONL), containing the cell bodies of rods and cones, which showed little oxidative enzyme activity in the sparse cytoplasm surrounding the nuclei of this layer. The outer plexiform layer (OPL) showed two zones of oxidative enzyme activity, with the outer two thirds being largely unreactive, apart from faint reaction products, probably associated with cone pedicles. The inner one third of the OPL was more strongly reactive, correlating with the dendritic processes of bipolar cells. The cell bodies of these bipolar cells make up the bulk of the inner nuclear layer (INL) which was only faintly reactive except on its border with OPL. The inner plexiform layer (IPL) was characterised by moderately high COX and SDH activity. In both oxidative enzyme reactions an intermediate zone of lower activity was often seen between the two sublaminae referred to as IPLa and IPLb. ${ }^{11}$ The retinal ganglion cell bodies (RG) showed moderate to high activity for both COX and SDH. The retinal nerve fibre layer (NFL) was uniformly reactive to both enzymes except where the layer was coursed by capillaries and larger vessels. These histological features and histochemical correlates are illustrated in Figure 2. ICC labelling of COX in the optic nerve and retina confirmed the localisation of the enzyme protein seen in the histochemical reactions.

\section{Discussion}

Histochemical assays provide an effective method of investigating mitochondrial enzyme activities. ${ }^{6}$ We have shown that this activity is preserved in human globes enucleated coincident with the cessation of vascular perfusion during multiple organ donation. This approach therefore lends itself for both the histochemical and biochemical characterisation of mitochondrial function in different ocular tissues.

The quantitative distribution of specific COX and SDH activities observed in human optic nerve and retina is in agreement with previous animal studies ${ }^{11} 12$ with the highest requirements for OXPHOS within the unmyelinated portion of retinal ganglion cells, the outer plexiform layer, inner segments of the photoreceptors, and the RPE. The RPE and retinal ganglion cells however appear to be particularly susceptible to the functional consequences of OXPHOS defects due to mtDNA mutations with retinal pigmentary changes and optic atrophy both being common ophthalmic manifestations of mitochondrial disorders. ${ }^{8}$

The pattern of oxidative enzyme activity within the retinal ganglion cells has implications for our understanding of the site of axonal dysfunction in those patients with mitochondrial optic nerve disease. Leber's hereditary optic neuropathy (LHON) is the archetypal optic neuropathy arising from mutations in mtDNA. ${ }^{5}$ It is characterised by maternally inherited acute or subacute bilateral visual loss and associated with the development of dense central or centrocaecal scotomas. Electrophysiological $^{13}$ and morphological studies $^{14}$ point towards a selective dysfunction of axons within the optic nerve as the underlying pathology in LHON. ${ }^{15}$ While the anatomical site of this process is unclear, the three primary LHON mutations all result in amino acid substitutions in different subunits of respiratory chain complex I causing disruption of OXPHOS. ${ }^{16}$ This suggests that the site of the lesion in LHON may in part be determined by reliance upon mitochondrial function.

The histochemical evidence indicates that the unmyelinated portion of the optic nerve has greater demands for mitochondrially derived ATP than the myelinated posterior segment. In neurons, mitochondrial oxidative phosphorylation accounts for over $95 \%$ of all ATP production of which approximately half is utilised by ATPase dependent ionic pumps essential for the transmission of action potentials. ${ }^{17}$ In myelinated fibres, conduction proceeds by depolarisation at successive nodes of Ranvier ${ }^{18}$ with clustering of pump ATPases only within the nodal regions. ${ }^{19}$ Depolarisation in unmyelinated lengths of axon is a more generalised phenomenon requiring a greater flow of curren $\mathrm{t}^{18}$ and is associated with a more uniform membrane distribution of pump ATPases. ${ }^{19}$ In dendrites and synaptic terminals, high rates of ion transport ${ }^{20}$ are associated with high levels of both ATPase ${ }^{21}$ and COX activity. ${ }^{22}$ Cumulatively, this suggests that the high levels of mitochondrial enzymes in the prelaminar and laminar regions of the human optic nerve are necessary to maintain transmission of action potentials along the unmyelinated portion of the axon.

A deficiency in mitochondrially derived ATP has been proposed as the proximate cause of ganglion cell death in LHON. ${ }^{23}{ }^{24}$ The distribution of $\mathrm{COX}$ and $\mathrm{SDH}$ suggests that any disruption in mitochondrial energy production is most likely to result in a functional lesion within the unmyelinated portion of the optic nerve. This is consistent with a recently proposed model for optic nerve degeneration in LHON based upon ganglion cell dysfunction due to failure of axoplasmic transport at the level of the lamina cribrosa. ${ }^{16}$

This study was funded by the Medical Research Council, Northern Regional Health Authority, and the Royal Victoria Infirmary Trustees, Newcastle upon Tyne. We thank the Northern Regional transplant coordinators for their help.

The authors have no commercial or proprietary interest in the products or equipment discussed in this article.

1 Mitchell P. Vectorial chemistry and the molecular mechanics of chemiosmotic coupling: power transmission by proticity. Biochem Soc Trans 1976;4:399-430.

2 Schapira AHV, DiMauro S, eds. Mitochondrial disorders in neurology. Oxford: Butterworth-Heinemann, 1994.

3 Brown GC. Control of respiration and ATP synthesis in mammalian mitochondria and cells. Biochem f 1992;284:113 . 
4 Taylor RW, Birch-Machin MA, Bartlett K, et al. The control of mitochondrial oxidations by complex III in rat muscle and liver mitochondria 7 Biol Chem 1994;269:3523-8.

5 Howell N. Primary LHON mutations: trying to separate

6 Old SL, Johnson MA. Methods of microphotometric assay of succinate dehydrogenase and cytochrome c oxidase for use on human skeletal muscle. Histochem f 1989;21:545-55.

7 Birch-Machin M, Jackson S, Singh Kler R, et al. Study of skeletal muscle mitochondrial dysfunction. Methods Toxicol 1993;2:51-69.

8 Newman NJ. Mitochondrial diseases and the eye. Ophthalmol Clin North Am 1992;5:405-24.

9 Johnson MA, Kadenbach B, Droste $M$, et al. Immunocytochemical studies of cytochrome oxidase subunits in skeletal muscle of patients with partial cytochrome oxidase deficiencies. 7 Neurol Sci 1988;87:75-90.

10 Sternberger LA. Immunocytochemistry. 2nd ed. New York: John Wiley, 1979.

11 Kageyama GH, Wong-Riley MTT The histochemical localization of cytochrome oxidase in the retina and lateral geniculate nucleus of the ferret, cat, and monkey, with pargeniculate nucleus of the ferret, cat, and monkey, with particular reference to retinal mosaics and

12 Lessel S, Horovitz B. Histochemical study of enzymes of optic nerve of monkey and rat. Am f Ophthalmol 1972;74:118-26.

13 Sherman J, Kleiner L. Visual system dysfunction in Leber's hereditary optic neuropathy. Clin Neurosci 1994;2:121-9.

14 Sadun AA, Kashima Y, Wurdeman AE, et al. Morphological findings in the visual system in a case of Leber's hereditary optic neuropathy. Clin Neurosci 1994;2:165-72.
15 Riordan-Eva P, Sanders MD, Govan GG, et al. The clinical features of Leber's hereditary optic neuropathy defined by the presence of a pathogenic mitochondrial DNA mutation. Brain 1995;118:319-37.

16 Howell N. Leber's hereditary optic neuropathy: how do mitochondrial DNA mutations cause degeneration of the optic nerve? F Bioenerg Biomembr 1997;29:165-73.

17 Erecinska M, Silver IA. ATP and brain function. 7 Cereb Blood Flow Metab 1989;9:2-19.

18 Smith KJ. Conduction properties in central demyelinated and remyelinated axons, and their relation to symptom production in demyelinating disorders. Eye 1994;8:224-37.

19 Waxman SG, Ritchie JM. Molecular dissection of the myelinated axon. Ann Neurol 1993;33:121-36.

20 Kadekaro M, Crane AM, Sokoloff L. Differential effects of electrical stimulation of sciatic nerve on the metabolic activity in spinal cord and dorsal root ganglion in the rat. Proc Natl Acad Sci USA 1985;82:6010-13.

21 Pech IV, Stahl WL. Immunocytochemical localization of $\mathrm{Na}+\mathrm{K}+$ ATPase in primary cultures of rat retina. Neurochem Res 1984;9:759-69.

22 Kageyama GH, Wong-Riley MTT. Laminar and cellular localization of cytochrome oxidase in the cat striate cortex. 7 Comp Neurol 1986;245:137-59.

23 Brown MD, Voljavec AS, Lott MT, et al. Leber's hereditary optic neuropathy: a model for mitochondrial neurodegenerative diseases. FASEB f 1992;6:2791-9.

24 Rizzo JF III. Adenosine triphosphate deficiency: a genre of optic neuropathy. Neurology 1995;45:11-16. 